\title{
Young Soccer Players With Higher Tactical Knowledge Display Lower Cognitive Effort
}

Perceptual and Motor Skills $0(0) \mathrm{I}-16$

(C) The Author(s) 2019 Article reuse guidelines: sagepub.com/journals-permissions DOI: $10.1177 / 0031512519826437$ journals.sagepub.com/home/pms

@SAGE

\author{
Felippe da S. L. Cardoso' (D, \\ Sixto González-Víllora ${ }^{2}$, José Guilherme ${ }^{3}$, \\ and Israel Teoldo'
}

\begin{abstract}
The present study aimed to investigate whether the form and amount of declarative tactical knowledge (DTK) and procedural tactical knowledge (PTK) influence cognitive effort during soccer performance among young players. We assessed 36 male players from a Brazilian first-division soccer club; participants averaged 14.89 $(S D=1.42)$ years of age. We evaluated DTK from video simulation tests and PTK through the System of Tactical Assessment in Soccer. We assessed cognitive effort by measures of pupil diameter using Mobile Eye Tracking-XG while players viewed soccer video scenes and made game-related play decisions. After the assessment of tactical knowledge, we categorized the sample according to players' tactical knowledge into participants with higher and lower PTK and higher and lower DTK. Subsequently, we examined the both PTK and DTK groups on cognitive effort. Our results suggest that tactical knowledge influences cognitive effort in that players with higher PTK and DTK displayed less cognitive effort during soccer performance tasks. In conclusion, we observed that PTK and DTK influenced the cognitive effort younger soccer players expended while viewing soccer scenes and making soccer performance decisions.
\end{abstract}

\footnotetext{
'Centre of Research and Studies in Soccer, Universidade Federal de Viçosa, Brazil

${ }^{2}$ University of Castilla-La Mancha, Spain

${ }^{3}$ University of Porto, Portugal

Corresponding Author:

Felippe da S. L. Cardoso, Núcleo de Pesquisa e Estudos em Futebol, Universidade Federal de Viçosa, Av. PH Rolfs, SN - Campus Universitário - Centro, Viçosa, Brazil.

Email: nupef.cardoso@gmail.com
} 


\section{Keywords}

pupillometry, cognition, decision-making, evaluation, sport psychology, tactical skills

\section{Introduction}

In the 1960s, research by Hess and Polt (1964) and Kahneman and Beatty (1966) identified pupillary behavior as a valid representation of cognitive effort used to perform a task. The pupil expands progressively as cognitive effort increases, and as cognitive effort is reduced, the pupil progressively returns to its baseline size (see also Johnson, Miller Singley, Peckham, Johnson, \& Bunge, 2014; Kahneman \& Beatty, 1966). Following these early findings, the mechanisms involved in the relation between pupillary behavior and cognitive effort have been extensively studied at broad anatomical and specific neural levels (Beatty \& Lucero-Wagoner, 2000; Reinhard \& Lachnit, 2002; Unsworth \& Robison, 2017).

Anatomically, it has been well established that changes in pupil size are mediated by the actions of two smooth muscles in the iris: the sphincter (or constrictor) and the dilator muscles of the pupil (Joshi, Li, Kalwani, \& Gold, 2016). The dilator muscle is under adrenergic control (sympathetic system) from the superior sympathetic ganglion (Steinhauer, Siegle, Condray, \& Pless, 2004). The pupil's sphincter muscle is innervated by cholinergic fibers from the parasympathetic system of the Edinger-Westphal nucleus (Steinhauer et al., 2004; Wilhelm, Wilhelm, \& Lüdtke, 1999). Through this anatomical-functional organization, the pupil reflects the activity of the nervous system by means of its response to activation of two types of neuromodulators (catecholamines and acetylcholine). Recent evidence indicates that the sympathetic autonomic control, modulated by the release of catecholinergic neuromodulators, makes it possible to measure cognitive effort during a task (for further details, see van der Wel \& van Steenbergen, 2018).

With respect to neural control, the mechanism of pupil excitation during cognitive effort is associated with the activation of specific neurons of the locus coeruleus (LC; Laeng, Sirois, \& Gredebäck, 2012; Sirois \& Brisson, 2014). The LC is a subcortical structure, and it is the conductor of the noradrenergic system (Schwarz \& Luo, 2015). The LC is connected to other regions of the brain responsible for specific levels of task demands, such as the anterior cingulate cortex. Anterior cingulate cortex efferent projections have an effect on the levels of neural gain along the cortex, including frontal and parietal influences on the cognitive control of the task (Aston-Jones \& Cohen, 2005; Nieuwenhuis, De Geus, \& Aston-Jones, 2011). However, other subcortical areas, such as the superior colliculus, are also important for information retrieval from stored memory (Sterpenich et al., 2006) and selective attention to the task at hand (Foote \& Morrison, 1987). The discovery that changes in pupil size 
correspond to activity in the LC and this chain of related brain functioning was essential to establishing pupillary behavior as an indicator of brain functioning and cognitive effort (Joshi et al., 2016).

In the context of this extensive research on pupillary behavior and its relation to cognitive effort, measures of pupillary behavior have gained notoriety and importance in several scientific fields (Beatty, 1982; Beatty \& Lucero-Wagoner, 2000; van der Wel \& van Steenbergen, 2018). For example, there have been investigations in such various different areas of cognitive effort as arithmetic reasoning in multiplication tasks (Hess \& Polt, 1964), reading and recalling patterns through memory (Kahneman \& Beatty, 1966; Sibley, Coyne, \& Baldwin, 2011), interference from environmental visual and sound stimuli (Laeng, Ørbo, Holmlund, \& Miozzo, 2011), successful and unsuccessful coding and decoding in memory evaluation (Papesh, Goldinger, \& Hout, 2012), cognitive processing of emotional stimuli (Bradley, Miccoli, Escrig, \& Lang, 2008), and attentional control (Alnaes et al., 2014), among others. In all the aforementioned studies, it has been clear that the lower cognitive effort required to perform a task, the better the individual's performance.

Despite the large number of studies using pupillary behavior to evaluate cognitive effort, we observed none using this method to assess cognitive effort within the context of soccer performance. The evaluation of cognitive effort in soccer is important, given the hard-to-predict nature of game play and its demands for a high number of cognitive skills and high cognitive effort for game-related problem-solving (Vickers \& Williams, 2017). The investigation of cognitive effort becomes relevant in soccer because the needs for cognitive resource use occur under high temporal pressure, which demands superior skills of the soccer players to make appropriate and timely decisions in a cognitively demanding environment (Vickers \& Williams, 2017). In addition to the factors described earlier, there are also technical, physical, and physiological requirements, which make the role of cognitive resources and their regulation by the soccer players even more burdensome (Alarcón, Castillo-Díaz, Madinabeitia, Castillo-Rodríguez, \& Cárdenas, 2018; Kearney \& Judge, 2017; Kunrath, Cardoso, Nakamura, \& Teoldo, 2018). For example, a player who receives a pass has fractions of seconds to (a) scan the environment (opponents and teammates' positioning, empty spaces, etc.); (b) define their possibilities of action (pass, etc.); (c) choose the best way to perform the action (at the motorbiomechanical-physiological plan); and, finally, (d) analyze the action outcome and make adjustments for possible subsequent actions. Therefore, the demands for cognitive resources during soccer matches can sometimes exceed optimal processing skills, resulting in elevated cognitive effort.

With respect to cognitive skills, past studies have suggested that tactical knowledge may influence the level of cognitive effort needed (Kannekens, ElferinkGemser, Post, \& Visscher, 2009; McPherson, 1993; Voss, Kramer, Basak, Prakash, \& Roberts, 2010; Williams, Davids, Burwitz, \& Williams, 1993). 
Many demands for time-restricted decisions during the game create high cognitive effort that may decrease player performance over time (Vickers \& Williams, 2017; Voss et al., 2010). In this context, good tactical knowledge enhances players' ability to meet cognitive effort requirements and, thus, respond better to the demands of the game (McPherson, 1993; Thomas \& Thomas, 1994).

It should be emphasized that the influence of tactical knowledge on cognitive effort should take into consideration the subdivisions of tactical knowledge (i.e., declarative and procedural) proposed by specialized literature (McPherson, 1993), as this subdivision implies different neural mechanisms and behaviors that are characteristic of each form of knowledge. McPherson (1993) points out that the mechanisms of action of these two forms of tactical knowledge are distinct, and this may impact how cognitive effort is influenced. Declarative tactical knowledge (DTK) is mainly associated with working memory and the ability to recognize and recall specific patterns. To be evoked by the players, this form of tactical knowledge must be processed explicitly, and this demands conscious information processing (Henke, 2010; McPherson, 1993). On the other hand, procedural tactical knowledge (PTK) is associated with the ability to perceive the situational demands of the game environment and perform, in advance, the appropriate game action. PTK is strictly linked to the automation of implicit perceptual-cognitive-motor processes without conscious player awareness (Henke, 2010; McPherson, 1993; Williams \& Davids, 1995).

Given the importance of tactical knowledge for in-game performance and the current paucity of scientific data demonstrating the relation of tactical knowledge and its forms (i.e., declarative and procedural) with cognitive effort, our interest was to examine whether tactical knowledge would be associated with cognitive effort and to verify whether the form and amount of DTK and PTK would influence cognitive effort.

Therefore, we sought to evaluate soccer players with respect to the amount of their PTK and DTK and then measure cognitive effort through an analysis of pupillary behavior in a laboratory-controlled performance task. We made associations to identify the influence of the amount and form (i.e., declarative and procedural) of tactical knowledge on cognitive effort and hypothesized that higher amounts of DTK and PTK would significantly and favorably influence (i.e., reduce) players' cognitive effort. Thus, we predicted that soccer players with higher PTK and DTK would display lower cognitive effort in relation to those with lower PTK and DTK.

\section{Method}

\section{Participants}

Thirty-six male academy soccer players from a Brazilian first-division soccer club with an average age of 14.89 years $(S D=1.42)$ participated in this study. 
As inclusion criteria, all players had to be engaged in regular soccer-specific training routines with at least five weekly sessions of 1.5 hours each and participating in national or international competitions. The time of players' deliberate practice in soccer was calculated from the record of the athletes in the club, with average values for the sample of $1,268.70$ hours $(S D=736.17)$. Before taking part in the research, all participants signed an informed consent. Participants younger than the age of 18 signed an assent agreement, and legal guardians of these younger participants signed the informed consent. All research procedures were in accordance with the norms established by the Resolution 466/2012 of the National Health Council and with the Declaration of Helsinki for human research. The project was approved by the Ethics Committee in Research with Human Beings of the Universidade Federal de Viçosa (No. 412.816-08/10/2013).

\section{Variables and Experimental Procedures}

PTK. To collect data regarding players' PTK, we used the System of Tactical Assessment in Soccer-FUT-SAT (Teoldo, Garganta, Greco, Mesquita, \& Maia, 2011). FUT-SAT allows the evaluation of players' PTK by analyzing their tactical actions with and without the ball during the performance task and calculating the percentage of their correct actions. FUT-SAT is based on the core tactical principles of soccer, considering five principles for the offensive phase and five principles of the defensive phase (for more information, see Teoldo et al., 2011).

The field test for this instrument was carried out on a field of 36 meters in length by 27 meters in width. To perform this test, participants were grouped into two teams, each with three outfield players and a goalkeeper (GK-3 vs. 3GK). Each team had a defender, a midfielder, and an attacker. We requested players to follow the official rules of the game. We gave players 30 seconds to become familiar with the test, and the test lasted four minutes, as recommended in an original protocol (Teoldo et al., 2011). The PTK test values are given in percent, varying between $0 \%$ and $100 \%$.

For the analysis of PTK, we followed the procedures proposed by Teoldo et al. (2011). Data analysis was performed by a trained evaluator after we demonstrated interrater reliability of $97 \%$ between two other evaluators. This is higher than the minimum reliability value proposed by the literature (Tabachnick \& Fidell, 2007).

DTK. To evaluate the players' DTK, we used a test protocol based on the players' analysis of game offensive situations presented on video scenes. This video test comprises 11 action scenes in actual soccer games with 11 players on each side, as projected on a screen, with a play duration of 5-13 seconds for each scene. Other experiments using this protocol have been published 
(Américo et al., 2017). During the experiment, we presented all 11 video sequences, pausing the actions and occluding the screen image prior to an action from the ball carrier (all the players who evaluated the scenes watched the scenes for the first time in these evaluations). Right after the video was paused, participants were instructed to verbally respond as quickly as possible to tell "what the ball carrier should do" at that moment. All participant responses were recorded through a microphone built into Mobile Eye Tracking-XG (Applied Science Laboratories, Bedford, MA, USA). After recording the test responses, the material was transcribed to digital format in Microsoft Word $^{\circledR}$, in a laptop computer (POSITIVE T model 3300 Intel Core $^{\mathrm{TM}}$ i3 processor). The transcribed response was analyzed and compared with a response to the same action scene by an official test panel (Mangas, 1999). If player responses agreed with the expert panel, we recorded a hit and awarded the player one point. Disagreements (errors) were not scored. The DTK test values are given in percent, varying between $0 \%$ and $100 \%$.

Cognitive effort. With regard to cognitive effort data, we used the pupillometry technique by recording pupil size continuously at a sampling rate of 60 times per second $(60 \mathrm{~Hz})$ using the Mobile Eye Tracking-XG (Applied Science Laboratories). The Mobile Eye Tracking is a device used to verify the mobile ocular tracking that allows measuring the dynamics of the individual's pupillary behavior through a system of cameras mounted in a pair of glasses. This equipment detects both the reflection of the pupil and the cornea, as determined by the reflection of an infrared light source on the surface of the cornea, displayed on a video image of the eye (Wilson, Vine, \& Wood, 2009). Pupil diameter (in millimeter) measurements were processed through the GazeTracker software (Applied Science Laboratories), allowing the pupil size measurement to be synchronized with the video task. The measurements of pupil diameter were later registered in Excel for Windows ${ }^{\circledR}$ 2016. Data lost due to subjects' blinking or head movements were excluded from analyses. No individuals or trials were eliminated due to excessive data loss.

We performed the assessment of cognitive effort through the players' pupillary behavior during the DTK experiment (see information on the structure of the video task in the earlier topic). The test was performed in a closed environment without external interference, such as sonorization, and we controlled luminosity, with variation smaller than 07 lux. After setting up the environment, the Mobile Eye Tracking-XG was adjusted, and we carried out the 9-point calibration procedure with the participants. We presented the video scenes of the test through a projector onto a retractable projection screen (TES-TRM $150 \mathrm{~V}$ with projection type "Matte White"), with measurements of $3.04 \times 2.28 \mathrm{~m}$. The video scenes were projected through the use of an HD projector (Epson Powerlite X14), mounted to the ceiling, with extended graphics array resolution of $2.0 \times 2.0$ meters. 
Participants were positioned standing 2.5 meters away from the screen. Before the start of the experimental task, we explained the procedures, and participants then performed rehearsals on two presented test scenes so as to ensure task familiarity. We periodically checked the calibration of the Mobile Eye Tracking-XG to ensure accuracy. This familiarization and calibration process lasted approximately 10 minutes for each participant, after which we started the test.

After the experimental protocol, we defined four different moments related to the presentation of the video for analysis of the pupillary behavior. These cuts sought to characterize different moments of information processing so as to indicate more precisely the relationship between tactical knowledge and cognitive effort. The first snippet for setting the baseline value of the pupil diameter was represented by $\mathrm{M} 0$, and this value was obtained from the lowest value of the pupillary diameter (PD) observed between the end of the calibration and the end of the experiment.

The other three evaluations were defined during the experimental protocol: (a) M1 Video was the phase in which the participant was watching the video; (b) M2 Verbalization was the stage in which the respondent was verbally responding to offer their game play decision; and (c) M3 Rest was the stage represented by the time interval after the participant's verbal response until the start of the next video scene.

\section{Data Analysis}

Following data collection, we divided the sample into two groups, according to the amount of DTK and PTK each participant demonstrated on the PTK and DTK tests. It is important to highlight that, for statistical analysis, the participants' classifications as higher and lower in tactical knowledge was separate and distinct for DTK and PTK. Thus, players within the higher DTK group were not necessarily the same as those in the higher PTK group (see Table 1).

Subsequently, taking into account the values of participant levels of DTK and PTK, we analyzed the measures related to cognitive effort, using each group's average values of PD, considered at each phase of the analysis (M0, M1, M2, and M3). For statistical purposes, we disregarded M0. We analyzed data distribution through the Shapiro-Wilk test, and we used one-way analysis of variance (ANOVA) to compare the groups for each of the three evaluative phases of the experiment (M1, M2, and M3). Following any significant ANOVA, we used Tukey's post hoc to examine statistical differences between each possible pair of the phases analyzed. We calculated the effect size for this analysis through the partial eta squared $\left(\eta \mathrm{p}^{2}\right)$, whose reference values are lower than 0.01 for low effect, between 0.02 and 0.06 for intermediate effect, and more than 0.14 for high effect (Levine \& Hullett, 2002).

To compare participant groups with higher and lower tactical knowledge, we used the $t$ test for independent measurements. For this analysis, we compared 
Table I. Descriptive and Inferential Values of the Groups With Higher and Lower Procedural and Declarative Tactical Knowledge.

\begin{tabular}{lllll}
\hline & Higher (TK) & & Lower (TK) & \\
\cline { 5 - 5 } Tactical knowledge & $M(S D)$ & & $M(S D)$ & $P$ \\
\hline Procedural tactical knowledge & $91.08(2.21)^{\mathrm{a}}$ & & $81.65(1.83)$ & $<.001$ \\
Declarative tactical knowledge & $78.27(7.09)^{\mathrm{a}}$ & & $53.55(7.55)$ & $<.001$ \\
\hline
\end{tabular}

Note. $\mathrm{TK}=$ tactical knowledge.

${ }^{\mathrm{a} S i g n i f i c a n t}$ differences between groups (higher and lower procedural tactical knowledge) in the MannWhitney test.

Significance level $p<.05$.

the variations in pupil diameter percentage values. Effect sizes were obtained from the value of the Cohen's $d$ whose reference values are insignificant effect $(d<0.19)$, small effect ( $d$ between 0.20 and 0.49 ), mean effect ( $d$ between 0.50 and 0.79 ), large effect ( $d$ between 0.80 and 1.29), and very large effect $(d>1.30$; Cohen, 1988; Rosenthal, 1996). Statistical procedures were performed through SPSS 24.0 software, and the level of significance was $p<.05$.

\section{Results}

One-way ANOVA results regarding PTK revealed significant differences in PD among the three moments (video, verbalization, and rest), regardless of the amount of PTK (lower PTK, $F(2)=131.450, p<.001, \eta_{\mathrm{p}}^{2}=0.81$; higher PTK, $\left.F(2)=42.135 p<.001, \eta_{\mathrm{p}}^{2}=0.62\right)$. It is possible to observe that the highest values of PD occurred at moments M1 Video (lower PTK, $M=7.15, S D=1.65$; higher PTK, $M=4.29, S D=1.52$ ) followed by M2 Verbalization (lower PTK, $M=3.13, S D=1.24$; higher PTK, $M=3.09, S D=1.39$ ) and M3 Rest (lower PTK, $M=1.73, S D=0.98$; higher PTK, $M=1.86, S D=1.04$ ). The same pattern was observed for DTK (lower DTK, $F(2)=168.776, p<.001, \eta_{\mathrm{p}}{ }^{2}=0.81$; higher DTK, $F(2)=98.70 p<.001, \eta_{\mathrm{p}}^{2}=0.72$ ). Tukey's post hoc indicated differences between all evaluated moments. We observed that the largest PD values also occurred at moments M1 Video (lower DTK, $M=7.01, S D=1.41$; higher DTK, $M=4.48, S D=1.08$ ), followed by M2 Verbalization (lower DTK, $M=4.82$, $S D=2.02$; higher DTK, $M=2.10, S D=0.66$ ) and M3 Rest (lower DTK, $M=1.87, S D=1.19$; higher DTK, $M=1.46, S D=0.60$ ).

Among the groups with different PTK levels, the $t$-test results point to significant differences among players only during M1 Video, $t(34)=5.402, p<.001$, $d=1.80$. For phases M2 Verbalization, $t(34)=0.728, p=.928, d=0.03$, and M3 Rest, $t(34)=-0.364, p=.7128, d=0.13$, no differences were observed. As for the DTK, the $t$-test results point to significant differences among players during the M1 Video, $t(34)=6.040, p<.001, d=2.01$, and M2 Verbalization, 


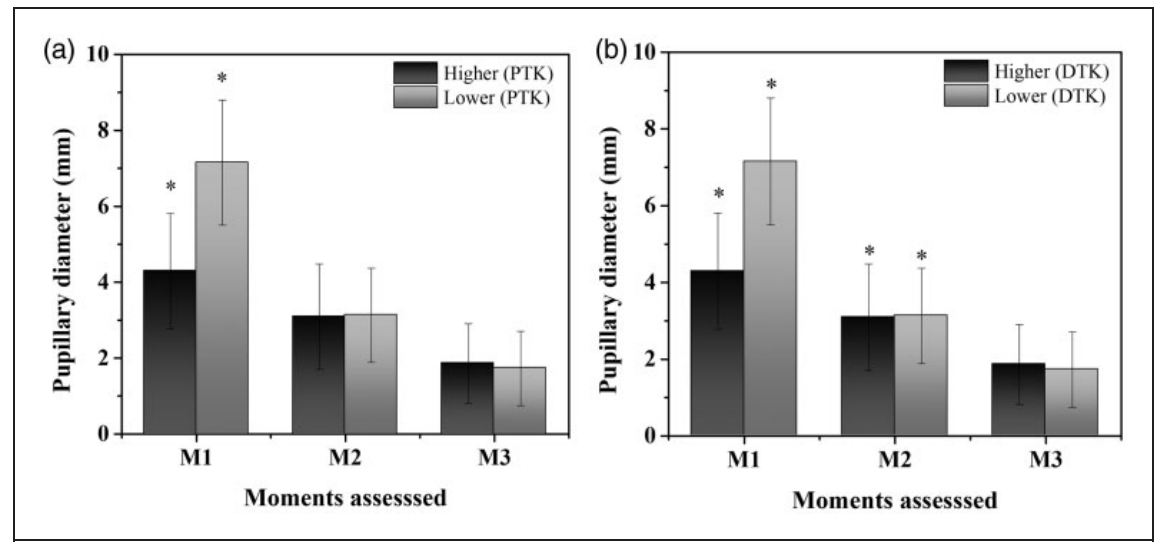

Figure I. Pupillary diameter (cognitive effort) of players with different levels of PTK and DTK in different moments of the test. MI-Video, M2-Verbalization, and M3-Rest. (a) Pupillary diameter (cognitive effort) of players with different levels of PTK in different moments of the test, and (b) pupillary diameter (cognitive effort) of players with different levels of DTK in different moments of the test. *Significant differences between the groups with higher and lower tactical knowledge.

DTK $=$ declarative tactical knowledge; PTK = procedural tactical knowledge.

$t(34)=5.436, p<.001, d=1.81$, with no significant difference in phase M3 Rest, $t(34)=1.284, p=.208, d=0.44$. For all significant differences found, players with higher DTK and PTK displayed lower cognitive effort during the evaluated phases. The results of cognitive effort of players with higher and lower PTK and DTK in the different phases of the test are presented in Figure 1.

\section{Discussion}

The aim of this work was to investigate whether tactical knowledge in soccer would be associated with cognitive effort during a soccer performance task while also verifying whether the form and amount of DTK and PTK would influence cognitive effort. The experimental results showed that tactical knowledge was associated with soccer players' cognitive effort such that players who possessed higher DTK and PTK required less cognitive effort to perform the soccer task.

Our findings indicate that, regardless of the form of tactical knowledge, the highest amount of cognitive effort in this soccer task occurred in phase M1 Video, the phase characterized by the player's need to process a series of game actions (teammates, opponents, and ball position, etc.) and then, based on the player's own cognitive and motor skills, quickly choose an optimal game response (Vickers \& Williams, 2017; Voss et al., 2010). These results are analogous to those by Kahneman and Betty (1966), who found a similar pattern in cognitive effort on a recognition and working memory recall test. In this study, 
the authors found that as task difficulty increased, cognitive effort also showed significant increases. The impact of cognitive effort on recognition and working memory recall tasks was replicated in several other studies (Klingner, Tversky, \& Hanrahan, 2011; Morris \& Jones, 1990), and this response pattern of cognitive effort has been observed in other areas, such as sustained attention processing (Laeng et al., 2011; Unsworth \& Robison, 2018), mental arithmetic (Hess \& Polt, 1964), and decision-making (Kahnemann \& Beatty, 1967). To our knowledge, ours is the first study to show this relationship between tactical knowledge and cognitive effort in soccer.

It is important to emphasize that in our study, players with higher DTK also displayed lower cognitive effort during the response or M2 Verbalization phase of the task. This result can be explained by the DTK structure, which allows the player a better conscious organization of the information captured during the task and, consequently, greater ease verbalizing a response (Henke, 2010; McPherson, 1993; Williams \& Davids, 1995). The present result may also be associated with the players' verbal skills (i.e., grammatical and lexicon facility), which may be related in turn to their level of academic instruction. As player verbal data were not collected, further research would be needed to investigate this possibility.

We observed small differences between DTK and PTK in their effects on cognitive effort. PTK influenced the perceptual-cognitive phase, M1 Video, while DTK also influenced the response verbalization phase, M2 Verbalization. These findings are reinforced by results of neuroimaging experiments showing that when individuals are engaged in tasks with demands of different forms of knowledge and evocation of information from the memory (declarative and nondeclarative), there are distinct behavioral responses (Henke, 2010; Squire, Kosslyn, Zola-Morgan, Haist, \& Musen, 1992). At neural levels, this difference may be associated with connections between structures of the medial temporal lobe (and, in particular, the hippocampus, along with the cingulate gyrus and the sublingual cortex) and conscious forms of memory representation associated with DTK (Moscovitch, 1995). As for PTK, the prefrontal, premotor, and motor cortex, as well as the striatum and mesencephalon (Shenhav, Botvinick, \& Cohen, 2013) and emotions controlled by the amygdala are more associated with intuitive aspects of motor behavior decision-making that accompany PTK (Kurzban, Duckworth, Kable, \& Myers, 2013). Therefore, due to these distinct characteristics at neural and behavioral levels, improvements in DTK and PTK tend to be associated with less cognitive effort during game situations (Kurzban et al., 2013; McPherson, 1993). For example, at neural levels, players with greater DTK or PTK may better use cognitive resources and show less activation of associated cortical areas, meaning that they use less cognitive effort. Findings supporting this possibility has already been observed in the literature (for further information, please see Naito \& Hirose, 2014). 
Thus, we can highlight the importance of improving DTK and PTK. According to González-Víllora, Serra-Olivares, Pastor-Vicedo, and da Costa (2015), better understanding of soccer through the improvement of tactical knowledge is learned and developed gradually throughout the training process. Consequently, the transfer of DTK to PTK is facilitated (Thomas \& Thomas, 1994; Williams \& Davids, 1995), and, concomitantly, PTK enables the assimilation and retention of DTK (Williams \& Davids, 1995). Therefore, each type of tactical knowledge facilitates understanding the other within a process known as proceduralization, which is the transition from DTK to PTK (Thomas \& Thomas, 1994; Williams \& Davids, 1995). This proceduralization plays a key role in the players' chances of achieving better performances (Williams \& Davids, 1995). Baumeister (1984) suggested that, in the sports' environment, when it is necessary to act under pressure, some athletes try to control their abilities consciously; however, through raised awareness of the action from the evocation of DTK, ironically, the chance of a successful performance is reduced due to divided attentional demand between the questions of What to do? and how to do it? (McPherson, 1993; Williams \& Davids, 1995). The player's need to consciously and explicitly evoke information may cause cognitive-temporal noise that interferes with the speed of actions in the context of the game when attention shifts instead to the quality of decision-making (van der Wel \& van Steenbergen, 2018; Vickers \& Williams, 2017). Thus, for high performance, the proceduralization process is crucial; it allows for automation of acquired tactical knowledge and nonconscious response selection follows (Williams \& Davids, 1995; Williams et al., 1993). In this way, the player gains significant time in the decision-making process. Based on our findings, there is then less cognitive effort expended in important phases of the performance task.

In practice, these research results indicate the importance of both DTK and PTK during the players' skill development. To achieve this, players must undergo a training process that is designed to represent real game situations that require decisions to understanding why these decisions are being made (Kannekens et al., 2009; Kearney \& Judge, 2017). Thus, the training process should not only enable players to develop tactical knowledge but should also allow proceduralization of this tactical knowledge to facilitate higher levels of PTK. These training elements will provide players with higher cognitive efficiency in their decision-making process during the game, as improved tactical knowledge (procedural and declarative) will mean that decision-making will occur with less cognitive effort. Thus, even with high physical and psychic game demands, players with more tactical knowledge may possess greater stored perceptual-cognitive potential, facilitating their cognitive effort.

It should be emphasized that this research represents a pioneer work in evaluating cognitive effort in soccer through the pupillometry technique. Further replication studies are needed, including the use of measures of cortical activation during performance tasks through electroencephalogram or 
functional magnetic resonance imaging. This additional information will help explain the mechanisms of neural control during assessments of cognitive effort and tactical knowledge. Other limitations that may be mentioned concern the less ecological character of the task we used to evaluate cognitive effort; we measured pupil response while players viewed videos and made decisions about soccer play rather than during actual play. Finally, our experimental design did not permit definitive causality inferences in the relationship between tactical knowledge and effort. However, this study demonstrated a strong relationship between young players' DTK and PTK and their reduced cognitive effort in game-related decision-making in soccer.

\section{Declaration of Conflicting Interests}

The author(s) declared no potential conflicts of interest with respect to the research, authorship, and/or publication of this article.

\section{Funding}

The author(s) disclosed receipt of the following financial support for the research, authorship, and/or publication of this article: The State Department of Sport and Youth of Minas Gerais in accordance with the State Act of Incentive to Sports, by FAPEMIG, CAPES, CNPQ, FUNARBE, the Dean's Office for Graduate and Research Studies, and the Centre of Life and Health Science at the Universidade Federal de Viçosa, Brazil, supported the study.

\section{ORCID iD}

Felippe da S. L. Cardoso (D) http://orcid.org/0000-0002-2861-6777

\section{References}

Alarcón, F., Castillo-Díaz, A., Madinabeitia, I., Castillo-Rodríguez, A., \& Cárdenas, D. (2018). La carga mental deteriora la precisión del pase en jugadores de fútbol [The mental load deteriorates the accuracy of the pass in soccer players]. Revista de Psicologia Del Deporte, 27(2), 155-164.

Alnaes, D., Sneve, M. H., Espeseth, T., Endestad, T., van de Pavert, S. H. P., \& Laeng, B. (2014). Pupil size signals mental effort deployed during multiple object tracking and predicts brain activity in the dorsal attention network and the locus coeruleus. Journal of Vision, 14(4), 1-20. doi:10.1167/14.4.1

Américo, H. B., Kowalski, M., Cardoso, F., Kunrath, C. A., González-Víllora, S., \& Teoldo, I. (2017). Difference in declarative tactical knowledge between U-11 and U-15 soccer players. Human Movement, 18(5), 25-30. doi:10.1515/humo-2017-0045

Aston-Jones, G., \& Cohen, J. D. (2005). An integrative theory of locus coeruleus-norepinephrine function: Adaptive gain and optimal performance. Annual Review of Neuroscience, 28(1), 403-450. doi:10.1146/annurev.neuro.28.061604.135709 
Baumeister, R. F. (1984). Choking under pressure: Self-consciousness and paradoxical effects of incentives on skillful performance. Journal of Personality and Social Psychology, 46(3), 610-620. doi:10.1037/0022-3514.46.3.610

Beatty, J. (1982). Task-evoked pupillary responses, processing load, and the structure of processing resources. Psychological Bulletin, 91(2), 276-292. doi:10.1037/00332909.91.2.276

Beatty, J., \& Lucero-Wagoner, B. (2000). The pupillary system. In J. T. Cacioppo, L. G. Tassinary \& G. G. Berntson (Eds.), Handbook of psychophysiology (2nd ed., pp. 142-162). New York, NY: Cambridge University Press Retrieved from http:// prx.library.gatech.edu/login?url=http://search.ebscohost.com/login.aspx? direct $=$ true $\& d b=$ psyh $\& A N=2000-03927-005 \&$ site $=$ ehost-live

Bradley, M. B., Miccoli, L. M., Escrig, M. A., \& Lang, P. J. (2008). The pupil as a measure of emotional arousal and automatic activation. Psychophysiology, 45(4), 602-607. doi:10.1111/j.1469-8986.2008.00654.x

Cohen, J. (1988). Statistical power analysis for the behavioral science. New York: Psychology Press.

Foote, S. L., \& Morrison, J. H. (1987). Extrathalamic modulation of cortical function. Annual Review of Neuroscience, 10(1), 67-95. doi:10.1146/ annurev.ne.10.030187.000435

González-Víllora, S., Serra-Olivares, J., Pastor-Vicedo, J. C., \& da Costa, I. T. (2015). Review of the tactical evaluation tools for youth players, assessing the tactics in team sports: Football. SpringerPlus, 4(1), 1-17. doi:10.1186/s40064-015-1462-0

Henke, K. (2010). A model for memory systems based on processing modes rather than consciousness. Nature Reviews Neuroscience, 11(7), 523-532. doi:10.1038/nrn2850

Hess, E., \& Polt, J. (1964). Pupil size in relation to mental activity during simple problem solving. Science, 140, 1190-1192. doi:10.1126/science.143.3611.1190

Johnson, E. L., Miller Singley, A. T., Peckham, A. D., Johnson, S. L., \& Bunge, S. A. (2014). Task-evoked pupillometry provides a window into the development of shortterm memory capacity. Frontiers in Psychology, 218, 1-8. doi:10.3389/fpsyg.2014.00218

Joshi, S., Li, Y., Kalwani, R. M., \& Gold, J. I. (2016). Relationships between pupil diameter and neuronal activity in the locus coeruleus, colliculi, and cingulate cortex. Neuron, 89(1), 221-234. doi:10.1016/j.neuron.2015.11.028

Kahneman, D., \& Beatty, J. (1966). Pupil diameter and load on memory. Science, 154, $1583-1586$.

Kahnemann, D., \& Beatty, J. (1967). Pupillary responses in a pitch-discrimination task. Perception \& Psychophysics, 2(3), 101-105. doi:10.3758/BF03210302

Kannekens, R., Elferink-Gemser, M. T., Post, W. J., \& Visscher, C. (2009). Self-assessed tactical skills in elite youth soccer players: A longitudinal study. Perceptual and Motor Skills, 109(2), 459-472. doi:10.2466/pms.109.2.459-472

Kearney, P. E., \& Judge, P. (2017). Successful transfer of a motor learning strategy to a novel sport. Perceptual and Motor Skills, 124(5), 1009-1021.

Klingner, J., Tversky, B., \& Hanrahan, P. (2011). Effects of visual and verbal presentation on cognitive load in vigilance, memory, and arithmetic tasks. Psychophysiology, 48(3), 323-332. doi:10.1111/j.1469-8986.2010.01069.x 
Kunrath, C. A., Cardoso, F., Nakamura, F. Y., \& Teoldo, I. (2018). Mental fatigue as a conditioner of the tactical and physical response in soccer players: A pilot study. Human Movement, 19(3), 16-22. doi:10.5114/hm.2018.76075

Kurzban, R., Duckworth, A., Kable, J. W., \& Myers, J. (2013). An opportunity cost model of subjective effort and task performance. Behavioral and Brain Sciences, 36(6), 661-679. doi:10.1017/S0140525X12003196

Laeng, B., Ørbo, M., Holmlund, T., \& Miozzo, M. (2011). Pupillary stroop effects. Cognitive Processing, 12(1), 13-21. doi:10.1007/s10339-010-0370-z

Laeng, B., Sirois, S., \& Gredebäck, G. (2012). Pupillometry: A window to the preconscious? Perspectives on Psychological Science, 7(1), 18-27. doi:10.1177/17456916 11427305

Levine, T. R., \& Hullett, C. R. (2002). Eta squared, partial eta squared, and misreporting of effect size in communication research. Human Communication Research, 28(4), 612-625.

Mangas, C. J. (1999). Conhecimento declarativo no futebol: Estudo comparativo em praticantes federados e não-federados, do escalão de sub-14 [Declarative knowledge in soccer: Comparative study in federated and non-federated practitioners, from the u14 level] (master's thesis, p. 98). Faculdade de Desporto da Universidade do Porto, Universidade do Porto, Porto.

McPherson, S. L. (1993). Knowledge representation and decision-making in sport. In J. L. Starkes \& F. Allard (Eds.), Cognitive issues in motor expertise (pp. 159-188). North Holland: Elsevier Science Publishers doi:10.1016/S0166-4115(08)61470-3

Morris, N., \& Jones, D. M. (1990). Memory updating in working memory: The role of the central executive. British Journal of Psychology, 81(81), 111-121.

Moscovitch, M. (1995). Recovered consciousness - A hypothesis concerning modularity and episodic memory. Journal of Clinical and Experimental Neuropsychology, 17(2), 276-290.

Naito, E., \& Hirose, S. (2014). Efficient foot motor control by Neymar's brain. Frontiers in Human Neuroscience, 8, 1-7. doi:10.3389/fnhum.2014.00594

Nieuwenhuis, S., De Geus, E. J., \& Aston-Jones, G. (2011). The anatomical and functional relationship between the P3 and autonomic components of the orienting response. Psychophysiology, 48(2), 162-175. doi:10.1111/j.1469-8986.2010. 01057.x

Papesh, M. H., Goldinger, S. D., \& Hout, M. C. (2012). Memory strength and specificity revealed by pupillometry. International Journal of Psychophysiology, 83(1), 56-64. doi:10.1016/j.ijpsycho.2011.10.002

Reinhard, G., \& Lachnit, H. (2002). The effect of stimulus probability on pupillary response as an indicator of cognitive processing in human learning and categorization. Biological Psychology, 60(2-3), 199-215. doi:10.1016/S0301-0511(02)00031-5

Rosenthal, J. A. (1996). Qualitative descriptors of strength of association and effect size. Journal of Social Service Research, 21(4), 37-59. doi:10.1300/J079v21n04_02

Schwarz, L. A., \& Luo, L. (2015). Organization of the locus coeruleus-norepinephrine system. Current Biology, 25(21), R1051-R1056. doi:10.1016/j.cub.2015.09.039

Shenhav, A., Botvinick, M. M., \& Cohen, J. D. (2013). The expected value of control: An integrative theory of anterior cingulate cortex function. Neuron, 79(2), 217-240. doi:10.1016/j.neuron.2013.07.007 
Sibley, C., Coyne, J., \& Baldwin, C. (2011). Pupil dilation as an index of learning. Proceedings of the Human Factors and Ergonomics Society, 55, 237-241. doi: $10.1177 / 1071181311551049$

Sirois, S., \& Brisson, J. (2014). Pupillometry. Wiley Interdisciplinary Reviews: Cognitive Science, 5(6), 679-692. doi:10.1002/wcs.1323

Squire, L. R., Kosslyn, S., Zola-Morgan, S., Haist, F., \& Musen, G. (1992). Memory and the hippocampus: A synthesis from findings with rats, monkeys, and humans. Psychological Review, 99(2), 195-231. doi:10.1037/0033-295X.99.3.582

Steinhauer, S. R., Siegle, G. J., Condray, R., \& Pless, M. (2004). Sympathetic and parasympathetic innervation of pupillary dilation during sustained processing. International Journal of Psychophysiology, 52(1), 77-86. doi:10.1016/ j.ijpsycho.2003.12.005

Sterpenich, V., D’Argembeau, A., Desseilles, M., Balteau, E., Albouy, G., Vandewalle, G.,... Maquet, P. (2006). The locus coeruleus is involved in the successful retrieval of emotional memories in humans. Journal of Neuroscience, 26(28), 7416-7423. doi:10.1523/JNEUROSCI.1001-06.2006

Tabachnick, B., \& Fidell, L. (2007). Using multivariate statistics (5th ed.). Needham Heights, Massachusetts, USA: Harper and Row Publishers.

Teoldo, I., Garganta, J., Greco, P., Mesquita, I., \& Maia, J. (2011). System of tactical assessment in soccer (FUT-SAT): Development and preliminary validation. Motricidade, 7(1), 69-84. doi:10.6063/motricidade.7(1).121

Thomas, K. T., \& Thomas, J. R. (1994). Developing expertise in sport: The relation of knowledge and performance. International Journal of Sport Psychology, 25(3), 295-312.

Unsworth, N., \& Robison, M. K. (2017). A locus coeruleus-norepinephrine account of individual differences in working memory capacity and attention control. Psychonomic Bulletin \& Review, 24(4), 1282-1311. doi:10.3758/s13423-016-1220-5

Unsworth, N., \& Robison, M. K. (2018). Tracking working memory maintenance with pupillometry. Attention, Perception, and Psychophysics, 80(2), 461-484. doi:10.3758/ s13414-017-1455-X

van der Wel, P., \& van Steenbergen, H. (2018). Pupil dilation as an index of effort in cognitive control tasks: A review. Psychonomic Bulletin and Review, 25(6), 1-11. doi:10.3758/s13423-018-1432-y

Vickers, J. N., \& Williams, A. M. (2017). The Role of Mental Processes in Elite Sports Performance. Oxford Research Encyclopedia of Psychology, 3(1), 1-25. doi:10.1093/ acrefore/9780190236557.013.161

Voss, M., Kramer, A. F., Basak, C., Prakash, R. S., \& Roberts, B. (2010). Are expert athletes 'expert' in the cognitive laboratory? A meta-analytic review of cognition and sport expertise. Applied Cognitive Psychology, 24, 812-826. doi:10.1002/acp.1588

Wilhelm, B., Wilhelm, H., \& Lüdtke, H. (1999). Pupillography: Principles and applications in basic and clinical research. In M. Böttcher \& J. Kuhlmann (Eds.), Pupillography: Principles, methods and applications (pp. 1-11). München, Germany: Zuckschwer.

Williams, M., \& Davids, K. (1995). Declarative knowledge in sport: A by-product of experience or a characteristic of expertise? Journal of Sport and Exercise Psychology, 17(3), 259-275. 
Williams, M., Davids, K., Burwitz, L., \& Williams, J. (1993). Cognitive knowledge and soccer performance. Perceptual and Motor Skills, 76(2), 579-593.

Wilson, M. R., Vine, S. J., \& Wood, G. (2009). The influence of anxiety on visual attentional control in basketball free throw shooting. Journal of Sport \& Exercise Psychology, 31, 152-168.

\section{Author Biographies}

Felippe da S. L. Cardoso has a Masters in physical education from the Universidade Federal de Viçosa. He is currently a PhD student in Physical Education at the Universidade Federal de Viçosa. Member of the Centre of Research and Studies in Soccer - NUPEF. He develops scientific works in the areas of Soccer focusing on the tactical component, the decision-making process, pupilometry and cognitive effort.

Sixto González-Víllora, PhD is an associate professor of Physical Education and Sports Pedagogy at the University of Castilla-La Mancha. Currently, he is Dean of the Faculty of Education in Cuenca (Spain) PhD. González-Víllora has conducted extensive research focusing on Pedagogical Models, Sports Assessment and Sporting Talent Detection Processes (tactical approach), Sports Training (perceptual, decision making and small sided-games) and other topics in Physical Education and Sports.

José Guilherme has a PhD in Sports Sciences from the Faculty of Sport at the University of Porto. He is an assistant professor of the Faculty of Sport of the University of Porto. He is the coordinator of the Soccer Office of the Faculty of Sport of the University of Porto, coach of the Portuguese Under20 Soccer Team, and a professor of Training Courses in Europe and South America. He is one of the world references in the study about the Tactical component in Soccer.

Israel Teoldo has $\mathrm{PhD}$ in Sports Sciences from the Faculty of Sport at the University of Porto and a Post-Doctor in Sports Science from Brunel University London. He is an associate professor of the Department of Physical Education at the Universidade Federal de Viçosa. He is also a coordinator of the Centre of Research and Studies in Soccer - NUPEF, coordinator of the Lato Sensu Post-graduate Course in Soccer at Federal Universidade Federal de Viçosa, and a professor of Training Courses at $\mathrm{CBF}$ and CONMEBOL. He develops numerous researches focusing on the tactical component of the game and the decision making process. 